\title{
Lidil
}

Revue de linguistique et de didactique des langues

43 | 2011

Le rapport au savoir dans les discours professionnels

\section{Construire une expertise dans et par les discours professionnels}

\section{Alain Rabatel et Nathalie Blanc}

\section{(2) OpenEdition}

1 Journals

Édition électronique

URL : http://journals.openedition.org/lidil/3100

DOI : 10.4000/lidil.3100

ISSN : 1960-6052

Éditeur

UGA Éditions/Université Grenoble Alpes

\section{Édition imprimée}

Date de publication : 30 mai 2011

Pagination : 5-10

ISBN : 978-2-84310-201-1

ISSN : $1146-6480$

\section{Référence électronique}

Alain Rabatel et Nathalie Blanc, "Construire une expertise dans et par les discours professionnels », Lidil [En ligne], 43 | 2011, mis en ligne le 30 novembre 2012, consulté le 22 septembre 2020. URL :

http://journals.openedition.org/lidil/3100 ; DOI : https://doi.org/10.4000/lidil.3100 


\title{
Construire une expertise dans et par les discours professionnels
}

\author{
Alain Rabatel et Nathalie Blanc*
}

Ce numéro apporte sa contribution à l'analyse de «la part langagière du travail» (Boutet, 2001), partant de l'idée que la sphère du travail n'est pas qu'un lieu de recueil de données, mais un moment stratégique où l'analyse des composants langagiers et communicationnels des situations de travail aide à la réalisation même de la tâche (EquoyHutin, 2009). Cette préoccupation, partagée par des approches en ergonomie (Clot, Faïta, Fernandez et Scheller, 2001), est également au cœur d'un certain nombre de travaux de linguistes (didacticiens, analystes du discours, interactionnistes) et de spécialistes des sciences de l'éducation (voir, entre autres, les publications dirigées par Guernier, DurandGuerrier et Sautot, 2006; Blanc et Varga, 2006; Filliettaz et SchubauerLeoni, 2008; Rabatel, 2004, 2010).

Les discours professionnels englobent, au-delà des écrits scientifiques (analysés dans Lidil $\mathrm{n}^{\circ} 41$ encore récemment), des discours de formation. Distincts des écrits académiques de recherche, ces discours de formation professionnelle sont très divers selon les cursus, les publics et les métiers. C'est cette diversité des discours professionnels qui fait l'objet des contributions de ce numéro, envisagée d'une part du point de vue des futurs professionnels ou du point de vue de leurs formateurs, d'autre part du point de vue des pratiques discursives professionnelles mises à contribution. Si les pratiques discursives professionnelles reposent souvent sur des usages conjoints de l'écrit et de l'oral (les mémoires donnent lieu à une soutenance, les visites formatives font l'objet de rapports écrits, etc.), elles côtoient aussi d'autres pratiques discursives auxquelles l'essor des nouvelles technologies numériques donne une importance croissante.

Les discours professionnels représentent des espaces de réflexion essentiels : lieu de consignation du savoir, ils sont aussi le lieu de la

* ICAR, Université Lyon 1, IUFM. 
construction de connaissances, de la confrontation entre des savoirs et des expériences concrètes, bref, à divers titres, ce sont des lieux où se dressent des constats, où se vérifient des hypothèses, des analyses savantes, où s'étayent des argumentations, des démonstrations. La maitrise de l'écriture de ces différents genres professionnels requiert non seulement celle des connaissances disciplinaires, mais aussi celle de stratégies d'argumentation appropriées et de techniques énonciatives pertinentes qui favorisent le positionnement de soi, la gestion des consensus ou des dissensus. Ce positionnement nécessite la prise en compte d'un certain nombre de spécificités, notamment celles de l'épistémologie des savoirs enseignés, celles des contextes socio-historico-culturels dans lesquels s'organisent les enseignements et les apprentissages et celles des conditions matérielles qui créent des contraintes sur ces discours « situés».

Ce numéro s'interroge centralement sur la manière dont ces discours permettent de construire une identité socio-discursive à travers la référence aux contenus scientifiques, ainsi qu'à travers leur regard sur les faits et les expériences professionnelles et la façon dont ils problématisent les uns et/ou les autres, notamment à travers leurs modes d'argumentation et de justification. L'objectif de ce numéro est ainsi d'analyser comment les écrits scientifiques et institutionnels s'inscrivent dans les discours professionnels, et, réciproquement, comment le locuteur/énonciateur se constitue en agent doté d'une autorité intellectuelle alliée à une expertise professionnelle. L'appel à contributions invitait à s'interroger sur le «rapport au savoir» et donc sur les conceptions du savoir, de sa transmission, de sa construction, sur la posture de l'auteur du discours professionnel et à tenter de répondre aux questionnements suivants :

- comment référer aux savoirs savants, aux savoirs institutionnalisés? Y a-t-il des domaines objectifs et d'autres qui sont réservés à l'expression de la subjectivité?

- comment faire acte de citation, comment gérer la polyphonie énonciative et la diversité des points de vue, dans l'optique de la construction d'une identité socio-discursive professionnelle?

Ces deux premières questions, qu'il était difficile d'évacuer de la réflexion, n'ont pourtant pas été au centre des analyses, afin de ne pas reconduire des recherches qui ont fait l'objet de plusieurs livraisons de Lidil; aussi a-t-on préféré mettre l'accent sur d'autres questions : 
- comment référer à l'expérience en ne se contentant pas de la décrire sous la forme d'un constat pur et simple, mais en la problématisant? Comment réfléchit-on (sur) cette expérience personnelle, comment la transforme-t-on en processus d'expertise en la mettant en relation avec des discours savants, des discours institutionnels?

- quel rapport entre des savoirs communs et des expériences singulières ou intersubjectives, notamment dans les cas, de plus en plus fréquents, de co-production des discours professionnels où la singularité de la voix de l'un se raffermit aussi dans le dialogue avec celle de l'autre?

- d'une façon plus générale, comment argumenter, quelles fonctions argumentatives accorde-t-on aux comptes rendus d'expériences, aux faits, aux données scientifiques?

Cette réflexion collective se décline dans le numéro à travers dix contributions diverses par :

- les objets d'étude : rapport de visite formative, récit de vie, mémoire professionnel, discours oraux en activité, etc.;

- les choix méthodologiques et cadres de référence : sciences du langage, sciences de l'éducation;

- les contextes professionnels observés : des enseignants experts ou débutants, des formateurs, des étudiants, des professionnels et des stagiaires dans des métiers techniques ou de la santé.

Cette diversité donne lieu à quatre sous-ensembles qui sont autant de facettes d'une expertise professionnelle en construction. Dans chacun d'eux, on a croisé le regard des spécialistes des sciences du langage et des sciences de l'éducation, tant il est apparu que ces perspectives sont complémentaires. Ces regards croisés ont notamment contribué à convoquer, décrire, voire théoriser le savoir sous ses formes multiples, telles qu’y fait référence le pluriel du titre du présent ouvrage : savoirs scientifiques, savoirs institutionnels, savoirs expérientiels, savoirs de conviction.

Les deux premières contributions, de chercheurs suisse et canadien, s'intéressent aux rapports aux savoirs de formateurs et de stagiaires en activité professionnelle, dans des métiers techniques pour le premier, de la santé pour la seconde. 
Laurent Filliettaz analyse le rapport au savoir dans des discours de formateurs dans le champ de la mécanique et notamment des formes spontanées et situées d'explicitation en rapport avec les activités en cours d'accomplissement.

Marty Laforest met en relief le double rapport au savoir de la stagiaire sage-femme qui est experte devant sa cliente mais qui, devant sa formatrice, reste une étudiante devant acquérir un élément important de l'expertise : la souplesse dans le maniement du savoir.

Les deux contributions suivantes analysent le rapport aux savoirs et la manipulation des savoirs dans la pratique enseignante.

Véronique Rivière et Lucile Cadet analysent deux discours professionnels de retour d'expérience par entretiens d'auto-confrontation. Elles mettent au jour par contraste la manière dont chaque enseignante mobilise ses savoirs professionnels pour interpréter une action d'enseignement.

Solveig Lepoire-Duc observe l'usage que deux enseignants font du tableau noir pour appliquer, en grammaire, une nouvelle démarche de conceptualisation. Elle étudie ainsi dans quelles mesures incorporer un nouveau geste professionnel à la pratique enseignante peut conduire à reconfigurer des routines.

Trois contributions analysent ensuite les représentations des savoirs en formation d'enseignants à travers le récit de vie, le rapport de visite et le rapport aux textes virtuels.

Frédéric Torterat tente de montrer dans quelle mesure les récits de vie, quand ils sont envisagés et pratiqués comme des discours professionnels, donnent un éclairage inédit sur les savoirs d'expérience, et permettent de reformuler le rapport aux savoirs et aux savoir-être.

Fabienne Rondelli et Anne Leclaire-Halté analysent la place des savoirs et des professeurs stagiaires dans les rapports de visite, un genre de discours professionnel caractérisé par de grandes variations rédactionnelles et par la pluralité de ses enjeux communicationnels.

Véronique Rey et Céline Beaudet traitent des rapports aux textes virtuels en formation de lettres et des mutations vers une nouvelle écriture professionnelle, plus réticulaire.

Enfin, trois contributions s'intéressent au rapport aux savoirs dans l'écriture de discours professionnels, soit en termes de construction identitaire, soit en termes de profil d'accompagnement.

Michel Lusetti, à l'aide des outils de l'analyse de discours et de la linguistique énonciative analyse le mémoire professionnel comme un 
écrit de formation réflexif qui permet à son auteur de se reconnaitre et d'être reconnu comme nouveau professionnel.

Nathalie Blanc, dans une perspective socio-cognitive, s'attache à décrire comment l'identité professionnelle se construit, dans et par l'interaction, pendant le processus de réflexion du mémoire en partant de l'hypothèse du conflit socio-cognitif.

Enfin, Gilles Leclercq et Anne-Catherine Oudart s'intéressent à l'accompagnement des écrits professionnels, aux postures, savoirs et convictions des enseignants. Ils proposent ainsi trois sensibilités épistémiques, affectives et axiologiques différentes.

La plupart des dispositifs explorés, qui concernent la formation des enseignants, font écho à l'époque d'avant la mastérisation. Ils soulignent que la construction d'une expertise professionnelle, qui ne peut se faire à la place des principaux intéressés, requiert l'aide des pairs, des tuteurs, de modèles de référence, et finalement d'allers et retours incessants entre connaissance et reconnaissance. Tout cela nécessite des espaces d'expérimentation, d'analyse des expériences, des moments de retours réflexifs sur les savoirs, donc des lieux et des modules de formation dans un schéma général d'alternance. Car il est vain de croire que la pratique se règle une fois que les compétences académiques seraient définitivement acquises...

\section{RÉFÉRENCES BIBLIOGRAPHIQUES}

Blanc N. et VARga R. (coord.) (2006) : Lidil, $\mathrm{n}^{\circ}$ 34, Rapport de stage et mémoire professionnel. Normes, usages et représentations, Grenoble, ELLUG.

Boch F. et RINCK F. (coord.) (2010) : Lidil, nº 41, Énonciation et rhétorique dans l'écrit scientifique, Grenoble, ELLUG.

Boutet J. (2001) : «La part langagière du travail : bilan et évolution», Langage et société, n ${ }^{\circ}$ 98, p. 17-42.

Clot Y., Faïta D., Fernandez G. et Scheller L. (2001) : «Entretiens en autoconfrontation croisée : une méthode en clinique de l'activité», Éducation permanente, $\mathrm{n}^{\circ} 146$ (1), p. 17-25.

Equoy-Hutin S. (coord.) (2009) : «Valeurs et enjeux des écrits de travail», Semen, $\mathrm{n}^{\circ}$ 28, Besançon, Presses universitaires de Franche-Comté.

Filliettaz L. et Schubauer-Leoni M.-L. (éds) (2008) : Processus interactionnels et situations éducatives, Bruxelles, De Boeck. 
Guernier M.-C., Durand-Guerrier V. et Sautot J.-P. (dir.) (2006) : Interactions verbales, didactiques et apprentissages, Besançon, Presses universitaires de Franche-Comté.

Rabatel A. (dir.) (2004) : Interactions orales en contexte didactique. Mieux (se) comprendre pour mieux (se) parler et pour mieux ( $\left.s^{\prime}\right)$ apprendre, Lyon, Presses universitaires de Lyon.

RABATEL A. (dir.) (2010) : Les reformulations pluri-sémiotiques en contexte de formation, Besançon, Presses universitaires de Franche-Comté. 